\title{
New titles at a glance
}

\author{
Germanium \\ Edited by Kazumi Wada and \\ Lionel C. Kimerling \\ WILEY 336PP. £105.00
}

Photonics and Electronics with

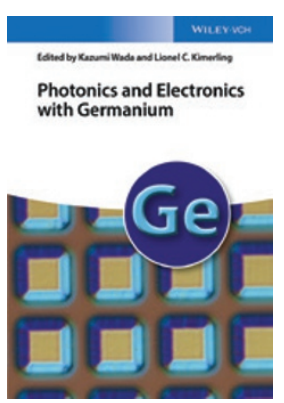

The first part of the book details the fundamental properties of germanium, such as band offsets, impurities, defects and surface structures, which determine the performance of germanium-

based devices in conjunction with conventional silicon technology. The second part covers methods of preparing and processing germanium structures, including chemical and physical vapour deposition, condensation approaches and chemical etching. The third and largest part gives a broad overview of the applications of integrated germanium technology: waveguides, photodetectors, modulators, ring resonators, transistors and, prominently, light-emitting devices. This book is an invaluable one-stop resource for both researchers and developers.

\section{Advanced Photon Counting}

Edited by Peter Kapusta, Michael Wahl and Rainer Erdmann

SPRINGER 384PP. US\$349.00

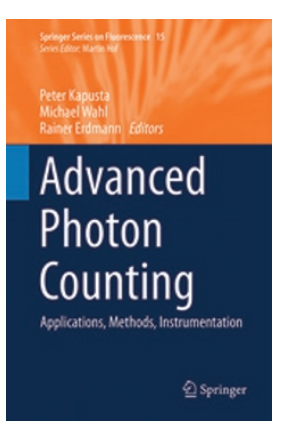

This volume focuses on timecorrelated single photon counting (TCSPC), a powerful tool allowing luminescence lifetime measurements to be made with high temporal resolution, even on single molecules. Several technologyrelated chapters present both the basics and current state-of-the-art, in particular of TCSPC electronics, photon detectors and lasers. The remaining chapters cover a broad range of applications and methodologies for experiments and data analysis, including the life sciences, defect centres in diamonds, super-resolution microscopy and optical tomography. The chapters detailing new options arising from the combination of classic TCSPC and fluorescence lifetime with methods based on intensity fluctuation represent a unique highlight.

\section{Efficient Organic Light Emitting Diodes (OLEDs)}

Edited by Yi-Lu Chang

PAN STANFORD PUBLISHING 126PP. US\$99.95

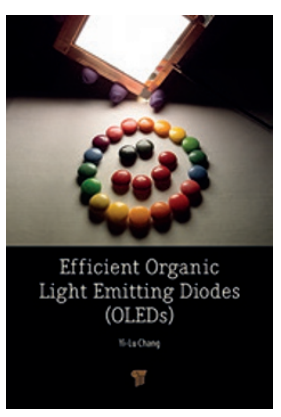

Organic lightemitting diodes (OLEDs) have steadily emerged as the ultimate display technology of choice for the coming decades. This book presents the knowledge needed for students and researchers

from diverse disciplines to understand the underlying principles of OLED technology. It contains a brief history and the fundamental working principles of OLEDs, more efficient OLED designs and advanced strategies to enhance performance.

Covered in detail are areas such as top emission, $\mathrm{p}$ - and n-type doping, device stability, light extraction and stacked white OLEDs. It also sheds light on the current industry practice and major areas of focus in the near future.

\section{Organic Electro-Optics and Photonics} By Larry R. Dalton, Peter Günter,

Mojca Jazbinsek, O-Pil Kwon and

\section{Philip A. Sullivan}

CAMBRIDGE UNIV. PRESS 300PP. $£ 49.99$

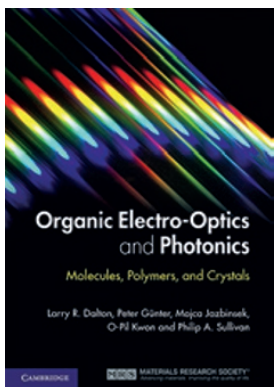

This text provides critical insight into recent advances in organic electrooptic materials, from the underlying quantum and statistical concepts through to the practical application of materials in modern devices and systems. It introduces theoretical and experimental methods for improving organic electro-optic and photonic technologies, reviews the central concepts of nonlinear optics, focusing on multi-scale theoretical methods and provides clear insight into the structure and function relationships critical to optimizing the performance of devices based on organic electro-optic materials. This book is a primer for the systematic nano-engineering of soft-matter materials and an invaluable resource for those involved in the development of modern telecommunication, computing, and sensing technologies depending on electro-optic technology. It also serves as a good reference for academic researchers and graduate students in the fields of chemistry, physics, electrical engineering, materials science and engineering, and chemical engineering.

\section{Introduction to Liquid Crystals for Optical Design and Engineering By Sergio R. Restaino and Scott W. Teare SPIE 130PP. US $\$ 44.00$}

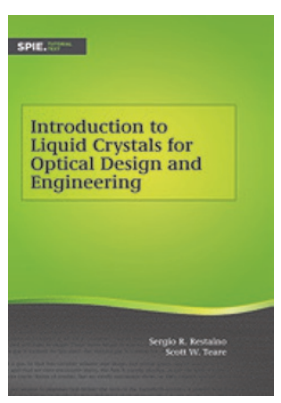

Liquid-crystal-based devices have become more popular for display technology used in mobile devices, vehicles, computer systems and almost any other opportunity for information display imaginable. Aiming to give the optics community a liquid crystals primer that focuses on the optical components made from these fascinating materials, this book provides a functional overview of liquid crystal devices, their history and their applications. Its nine chapters cover an overview of the properties of liquid crystals, the optical properties of polarization and birefringence as they apply to liquid crystals, as well as the use of liquid crystals in displays, spatial light modulators as optical element emulators and adaptive optics systems, and the future uses of liquid crystals. A historical overview of the literature on liquid crystals is provided in chapter 9 , which includes a chronological list of relevant publications, starting from 1888 . 